\section{Charging up MS treatment}

\section{By Lauren Martz, Staff Writer}

Researchers at MediGene AG have identified cationic liposomes that potentially could function as drug delivery vehicles or diagnostics tools for neuroinflammatory diseases such as multiple sclerosis. ${ }^{1}$ The company has data showing that the liposomes specifically target the inflamed blood brain barrier, although it's still unknown whether the delivery system can improve the safety or activity of MS therapeutics.

The preferential absorption of liposomes in specific tissues has been attributed to increased permeability of the capillaries supplying both tumor and inflamed tissue types, which can allow increased liposome diffusion. ${ }^{2}$

Cationic liposomes are known to specifically bind angiogenic endothelial cells. Thus, they initially were employed as tumor-targeting agents formulated with cancer therapeutics. ${ }^{3}$ Indeed, MediGene's EndoTAG-1 is a cationic liposome formulation of i.v. paclitaxel that has completed Phase II testing to treat inoperable, advanced or metastatic prostate cancer.

In a paper published in Molecular Pharmaceutics, Heinrich Haas and company colleagues now suggest that cationic liposomes could be used to target angiogenesis associated with acute inflammation, such as that of the spinal cord in MS patients.

Haas is associate director at MediGene and lead author on the paper.

In rats with experimental autoimmune encephalomyelitis (EAE), an animal model of MS, positively charged liposomes injected into the tail vein accumulated at the damaged blood brain barrier (BBB), as shown using laser scanning confocal microscopy.

Cationic liposome binding to the spinal cord of EAE rats was directly correlated with lower severity of disease and inflammation. In addition, the binding was detected even before the rats showed real signals of EAE, suggesting that the liposomes could prevent early changes in the neuroinflammatory process.

In contrast, the positively charged liposomes did not bind the spinal cords of healthy rats, and negatively charged liposomes did not bind to the spinal cords of either healthy or diseased rats.

"It will be interesting to see what happens when they actually use it to deliver drugs," said Simon Jones, VP of biology and ADMET at Epix Pharmaceuticals Inc. "The next step is to incorporate a drug known to work in MS into the liposomes and to use it in the EAE model to see if it can reduce inflammatory response. It could have been an extremely strong paper had they taken it this one step further."
Epix’s EPX-102216, an oral CC chemokine receptor 2 (CCR2) antagonist, is in preclinical testing to treat MS.

\section{Targeting for safety}

If therapeutics delivered via liposomes do show efficacy, the hope is that the compounds would have fewer side effects because of the more targeted delivery. In addition, the delivery method could be used to rescue compounds that were previously considered too toxic.

Haas told SciBX that "the local concentration of active agents at the sites of the affected blood brain barrier can be improved with respect to systemic administration," via cationic liposomes. The selective action of the active agent at these sites "will enable lower doses of drugs compared to systemic administration to reduce side effects or antibody production," he added.

Jones also noted that liposomes are passive targeting agents-they don't have surface elements that bind specific receptors, which can complicate the design of other types of carriers.

Haas did caution that the cationic lipids won't offer a cookie-cutter approach to formulating therapeutics. "The technology of loading/encapsulating an active compound to the liposome has to be selected on the basis of the molecular properties of the compound," he said.

Jones also said the large size of liposomes could be an issue. The molecules are in the range of " 10 to 100 s of nanometers. They will be taken up by the reticuloendothelial system in the liver and spleen, which can lead to toxicity of its own," he said. "The toxicity of the liposome-drug constructs will have to be compared with that of the drugs alone."

The authors of the Molecular Pharmaceutics article also suggested that cationic liposomes might have diagnostic applications.

"Cationic targeting offers determination of inflammatory events with a mechanism that does not depend on elevated blood brain barrier permeability," said Haas. Although increased permeability of the BBB is a characteristic of MS, it is not the first change associated with the disease.

Haas added that labeling cationic liposomes for in vivo imaging, such as MRI contrast, could be a suitable way to use them for diagnostic applications.

MediGene is not pursuing use of the cationic liposomes in MS. The patent status of the findings is undisclosed.

Martz, L. SciBX 2(15); doi:10.1038/scibx.2009.606

Published online April 16, 2009

\section{REFERENCES}

1. Haas, H. et al. Mol. Pharm.; published online March 12, 2009; doi:10.1021/ $\mathrm{mp} 8001478$

Contact: Heinrich Haas, MediGene AG, Martinsried, Germany e-mail: h.haas@medigene.com

2. Maeda, H. et al. Bioconjug. Chem. 3, 351-362 (1992)

3. Campbell, R. et al. J. Pharma. Sci. 98, 411-429 (2009)

COMPANIES AND INSTITUTIONS MENTIONED

Epix Pharmaceuticals Inc. (NASDAQ:EPIX), Lexington, Mass. MediGene AG (Xetra:MDG), Martinsried, Germany 